\title{
$S$-Nitrosation and Ubiquitin-Proteasome System Interplay in Neuromuscular Disorders
}

\author{
Salvatore Rizza, ${ }^{1}$ Costanza Montagna, ${ }^{2}$ Giuseppina Di Giacomo, ${ }^{2}$ \\ Claudia Cirotti, ${ }^{1}$ and Giuseppe Filomeni ${ }^{1,2}$ \\ ${ }^{1}$ Department of Biology, University of Rome "Tor Vergata", 00133 Rome, Italy \\ ${ }^{2}$ Research Center, IRCCS San Raffaele Pisana, 00166 Rome, Italy \\ Correspondence should be addressed to Giuseppe Filomeni; filomeni@bio.uniroma2.it
}

Received 24 May 2013; Revised 18 November 2013; Accepted 21 November 2013; Published 30 January 2014

Academic Editor: Alessio Cardinale

Copyright (C) 2014 Salvatore Rizza et al. This is an open access article distributed under the Creative Commons Attribution License, which permits unrestricted use, distribution, and reproduction in any medium, provided the original work is properly cited.

Protein $S$-nitrosation is deemed as a prototype of posttranslational modifications governing cell signaling. It takes place on specific cysteine residues that covalently incorporate a nitric oxide (NO) moiety to form $S$-nitrosothiol derivatives and depends on the ratio between NO produced by NO synthases and nitrosothiol removal catalyzed by denitrosating enzymes. A large number of cysteine-containing proteins are found to undergo $S$-nitrosation and, among them, the enzymes catalyzing ubiquitination, mainly the class of ubiquitin E3 ligases and the 20S component of the proteasome, have been reported to be redox modulated in their activity. In this review we will outline the processes regulating $S$-nitrosation and try to debate whether and how it affects protein ubiquitination and degradation via the proteasome. In particular, since muscle and neuronal health largely depends on the balance between protein synthesis and breakdown, here we will discuss the impact of $S$-nitrosation in the efficiency of protein quality control system, providing lines of evidence and speculating about its involvement in the onset and maintenance of neuromuscular dysfunctions.

\section{Redox Modifications and Cell Signaling}

The main molecular mechanism underlying signal transduction in eukaryotic cells relies on the regulation of protein function by posttranslational modifications. The transient and reversible attachment of reactive moieties on specific residues is able to induce a plethora of effects on protein function, thereby giving rise to a dynamic interplay of protein interactions capable to convey signals within the cell. Among these, redox signal is highly specific and represents a prerogative of sulfur-containing residues. In particular, cysteine is more versatile than methionine in forming adducts because of its capability to be present under numerous oxidation states [1]. This feature allows cysteine reacting with many oxidant species, such as hydrogen peroxide, glutathione disulfide (GSSG), or nitric oxide (NO) moiety, generating in such a way the reversible $S$-hydroxylated $(\mathrm{SOH}), S$ glutathionylated (SSG), or S-nitrosated (SNO) derivative, respectively [2]. Among them, glutathionylation or, widely, disulfide bond formation is the most stable cysteine oxidative modification. This is the reason why, physiologically, both $S$-hydroxylated and $S$-nitrosated proteins usually convert in $S$-glutathionylated (in the presence of high concentrations of GSH, as normally occurs inside the cells) or, widely, disulfide adducts. Therefore, hydrogen peroxide and NO can indirectly induce cysteine oxidation to disulfide. It should be also reminded that, similarly to hydrogen peroxide, NO overproduction has been reported being associated with irreversible sulfhydryl oxidation, such as sulfinylation $\left(\mathrm{SO}_{2} \mathrm{H}\right)$ or sulfonylation $\left(\mathrm{SO}_{3} \mathrm{H}\right)$ of metalloproteases [3]. However, these last modifications likely imply the production of peroxinitrite $\left(\mathrm{ONOO}^{-}\right)$, a more dangerous and more oxidant reactive nitrogen species (RNS) generated by the reaction between NO and superoxide anion $\left(\mathrm{O}_{2}{ }^{--}\right)$, which has been copiously reported being involved in protein tyrosine nitration (see below). 


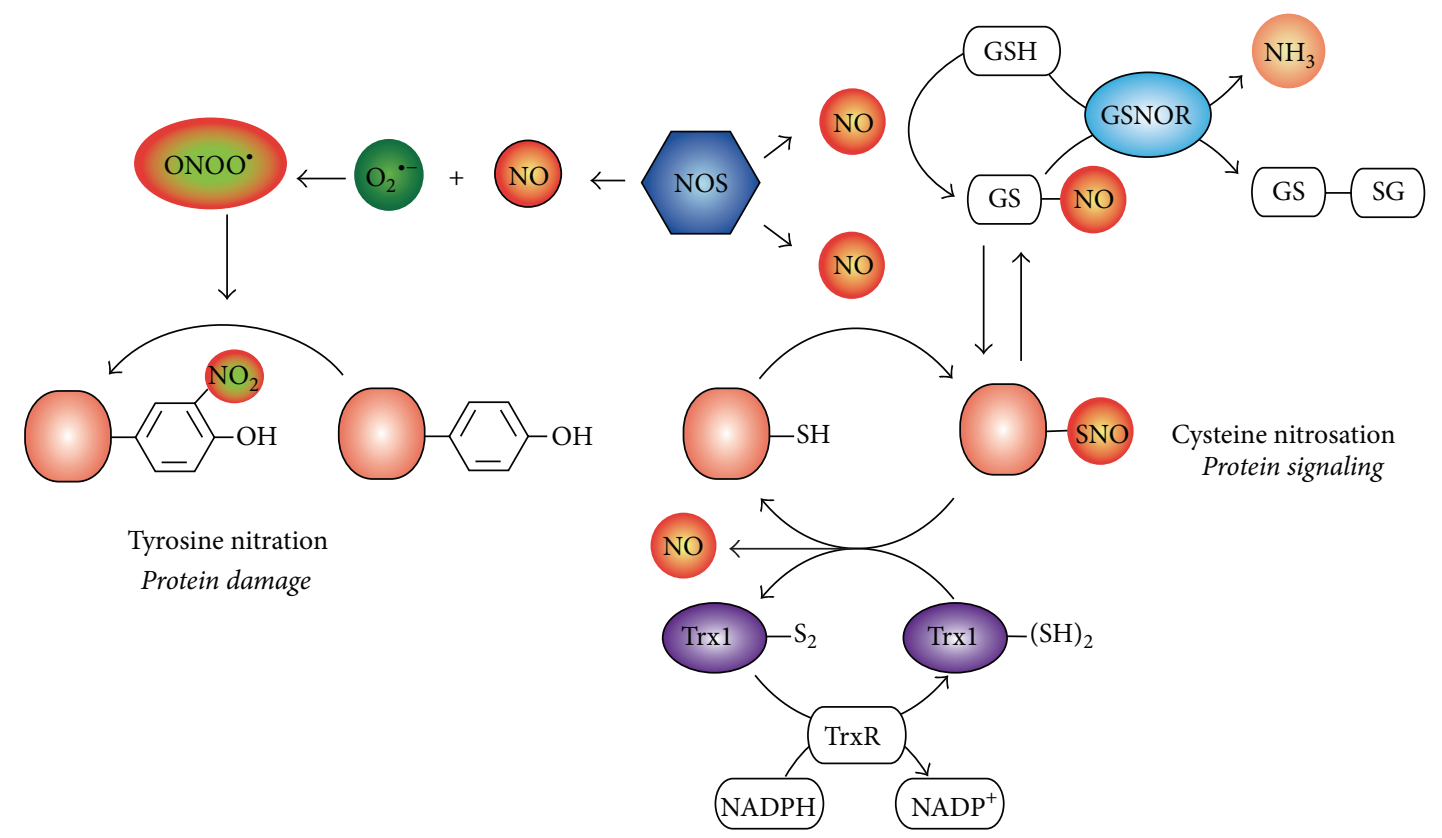

FIGURE 1: Tyrosine nitration versus $S$-nitrosation. Nitric oxide (NO), produced by NO synthase (NOS), can affect protein structure and function in different ways. Here only posttranslational modifications directly modifying protein residues are shown, tyrosine nitration (right) and cysteine $S$-nitrosation (left). The former adduct is irreversible (so far, no denitrating enzyme has ever been found) and responsible for protein damage occurring mostly upon the overproduction of NO. Indeed, under this condition (called nitrosative stress) NO can rapidly react with superoxide anion $\left(\mathrm{O}_{2}{ }^{--}\right)$to form peroxinitrite $\left(\mathrm{ONOO}^{-}\right)$which is the main harmful radical species inducing tyrosine nitration. Conversely, upon physiological production of NO, reactive cysteines of both redox-sensitive proteins and glutathione (GSH) can undergo $S$-nitrosation, thereby generating their $S$-nitrosothiol derivatives, Prot-SNOs and S-nitrosoglutathione (GSNO), respectively. Prot-SNOs and GSNO are in equilibrium by transnitrosation reactions; therefore, the GSNO catabolizing enzyme, GSNOR reductase (GSNOR), by regulating GSNO levels also impacts on protein nitrosation extent. Thioredoxin 1 (Trxl) also participates in protein denitrosation by means of its vicinal thiols that reduce Prot-SNO and oxidize to an internal disulfide bridge, whose further reduction is catalyzed by Trx reductase (TrxR) and ensured by reducing equivalents provided by NADPH. Although both GSNOR and Trxl concur to modulated protein $S$-nitrosation, it should be reminded that the former enzyme completely reduces GSNO to glutathione disulfide (GSSG) and ammonia $\left(\mathrm{NH}_{3}\right)$, whereas the latter releases the NO moiety of Prot-SNOs as NO itself or nitroxyl anion (HNO), which are species still capable to target protein substrates.

1.1. Implication of $\mathrm{NO}$ in Cell Signaling. Nitric oxide is a gaseous and membrane-diffusible radical molecule produced by the class of NADPH-dependent enzymes NO synthase (NOS) $[4,5]$. The first evidence of NO involvement in signal transduction goes back to 1983 when it was demonstrated that cGMP-mediated regulation of blood vessel tone depended on the direct binding of NO to the heme iron (Fe-nitrosylation) of guanylyl cyclase $[6,7]$. Since then, increasingly data provided further and indisputable lines of evidence pointing out this pathway being, in fact, implicated in many other functions, such as immune response [8], neurotransmission [9], and mitochondrial respiration [10]. Indeed, physiologically $\mathrm{NO}$ can bind to free iron within any heme-containing protein with a free ligand position [11]. By this reaction, and also by binding the copper binuclear centers in a noncompetitive manner [12], NO can regulate cytochrome $c$ oxidase activity and, more widely, it can tune mitochondrial respiration.

Alongside these findings, the involvement of $\mathrm{NO}$ in redox signaling was emerging and progressively assuming distinctive signatures. Over those years, it became clear, indeed, that $S$-nitrosation is the main reversible posttranslational modification induced by $\mathrm{NO}$ able to regulate different classes of proteins [13-15]. The discovery of denitrosating enzymatic systems, namely, those dependent on thioredoxin 1 (Trx1) and $S$-nitrosoglutathione reductase (GSNOR) activities, which actively participate the $S$-nitrosothiol-to-sulfhydryl (SNOto-SH) reduction [16-19] (Figure 1), definitively sealed the importance of $S$-nitrosation in cell physiology and human health (Table 1).

Actually, NO can also trigger irreversible modifications to proteins, such as the formation of nitrotyrosine. However, this is an event occurring only under certain conditions (nitrosative stress) that do not deal with signaling but just represents marker of damage. In particular, when $\mathrm{NO}$ is overproduced and no longer neutralized, it can react with oxygen-derived radical and nonradical species (ROS) thereby generating more dangerous RNS, for example, $\mathrm{ONOO}^{-}[20$, 21] (Figure 1).

As previously mentioned, protein $S$-nitrosothiols (PSNOs) generation is specific, redox-mediated, and reversible [22] depending on several factors, such as the environmental hydrophobicity conditions and the steric hindrance, as well as the net charge and the presence of oxygen $[2,13]$. In addition, it has been reported that the specificity of $S$-nitrosation can also depend on the presence of an $S$-nitrosation motif [23] roughly characterized by 
TABLE 1: Examples of pathological conditions associated with alterations in Prot-SNOs.

\begin{tabular}{lcc}
\hline Protein-SNO & Pathology & Reference \\
\hline Dynamin-related protein 1 & Alzheimer disease & Cho et al., 2009 [86] \\
Protein disulfide isomerase & Alzheimer disease & Uehara et al., 2006 [98] \\
& Parkinson disease & Nakamura et al., 2010 [30] \\
X-linked inhibitor of apoptosis & Alzheimer disease & Chung et al., 2004 [53] \\
Parkin & Parkinson disease & Fang et al., 2007 [97] \\
Peroxiredoxin-2 & Parkinson disease & Gonzalez et al., 2007 [99] \\
Ryanodine receptor 2 & Parkinson disease & Wei et al., 2010 [62] \\
$O^{6}$-alkylguanine-DNA alkyl transferase & Heart failure & Bellinger et al., 2009 [76] \\
Ryanodine receptor 1 & Cancer & Andersson et al., 2012 [100] \\
\hline
\end{tabular}

acid-base residues surrounding the target cysteine [24]. Nevertheless, many other factors deeply impact on the identity of specific proteins undergoing $S$-nitrosated, such as cellular localization and compartmentation of $\mathrm{NO}$ production [25]. In addition, the evidence that NO moiety can be also transferred among proteins or low-molecular-weight $S$-nitrosothiols (e.g., $S$-nitrosoglutathione, GSNO), adds further complexity to the regulation of protein $S$-nitrosation [26].

1.2. Transnitrosation and Denitrosation. The major determinant for NO transfer (transnitrosation) is the difference between the redox potential of the two interacting cysteine residues [27]. This aspect takes more importance if transnitrosation does not occur with low-molecular-weight thiols, but takes place between proteins. Indeed, this reaction is responsible for the propagation of many $\mathrm{NO}$-mediated cell signaling pathways and its significance has been highlighted quite recently in many physiopathological processes, such as (i) NO exchange between hemoglobin and the anion exchanger 1, which mediates NO release from erythrocytes [28]; (ii) transnitrosation among thioredoxin (Trx), caspase3 and the inhibitor of apoptosis (IAPs) proteins, which is involved in the regulation of cell death by apoptosis [27, 29-31]; (iii) glyceraldehyde 3-phosphate dehydrogenase (GAPDH)-mediated $S$-nitrosation of nuclear proteins, which contributes to cell death and accounts for the pathogenesis of several neurodegenerative diseases [32, 33]; (iv) transnitrosation between the neuronal-specific cyclin dependent kinase $5(\mathrm{Cdk} 5)$ and dynamin-related protein 1 (Drp1), which plays a pivotal role in mitochondrial dysfunction typical of neurodegenerations $[26,34]$.

PSNOs levels are counterbalanced by denitrosation systems, the most important of which are the glutathione (GSH)/GSNOR and the Trxl/Trx reductase (TrxR) couples $[2,18,35,36]$ (Figure 1). In the light of what mentioned above, only a specific subset of proteins is $S$-nitrosated, resulting in the selective modulation of specific signaling pathways. In this scenario, it is plausible that the propagation or modulation of cell signals by $S$-nitrosation often implies a crosstalk with signaling modalities mediated by other mechanisms of posttranslational modification [37]. In the last decades, the discovery of $S$-nitrosation of both protein kinases and phosphatases suggested the influence of this modification in a wide range of signal transduction pathways mediated by phosphorylation/dephosphorylation [38-40]. This aspect is of great importance if one considers that $S$-nitrosation can convert into $S$-glutathionylated/disulfide adduct and that this is a well-known mechanism driving signal transduction mediated by phosphorylative cascades [41]. Likewise, it has emerged that $S$-nitrosation may also operate in the nucleus on epigenetic mechanisms of transcriptional regulation, in particular by interfering with histone acetylation status [42]. Ubiquitination of proteins, which represents the pivotal reaction underlying protein turnover and quality control, might be also affected by $S$-nitrosation because many enzymes involved in ubiquitination process have critical cysteines, which have been reported undergoing oxidation [43-45] or, actually, sumoylation [46]. S-nitrosation could therefore have deep implications in a number of pathophysiological conditions involving ubiquitin-proteasome system (UPS).

\section{Dual Role of $S$-Nitrosation in Ubiquitin-Proteasome System}

2.1. Ubiquitination. Ubiquitination is an enzymatic posttranslational modification process occurring on proteins, based on the ligation of ubiquitin (an $8.5 \mathrm{kDa}$ protein) on a target protein lysine residue (monoubiquitination). This first step may be followed by the formation of ubiquitin chains through the attachment of additional ubiquitin moieties to one or more of the seven lysine residues within conjugated ubiquitin (polyubiquitination). The covalent attachment of ubiquitin on lysine residues requires the coordinated reaction of three enzymes. The first reaction is accomplished by the ubiquitin-activating enzymes (E1), which promote ubiquitin adenylation required for its covalent binding to the cysteine residue located at the $\mathrm{E} 1$ active site. Ubiquitin-charged E1 enzymes next transfer ubiquitin to the cysteine of the ubiquitin-conjugating enzymes (E2) that, in concert with a wide class of enzymes known as ubiquitin ligases (E3) needed for target recognition, finally transfer ubiquitin on lysine residues of specific substrates [47]. Protein ubiquitination is the major mechanism underlying protein turnover and 


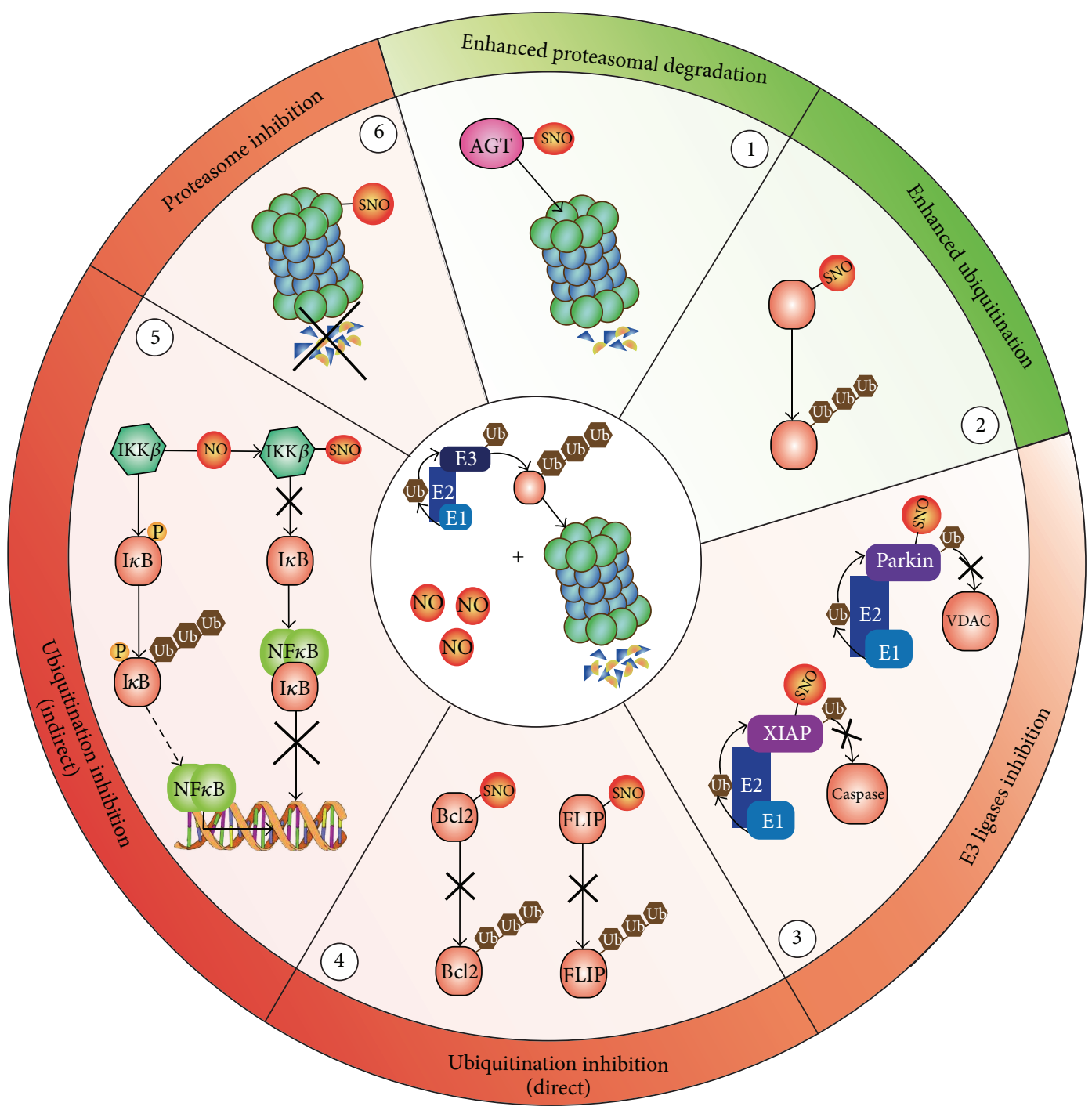

FIGURE 2: S-nitrosation-induced cellular effects on ubiquitin-proteasome system. Nitric oxide (NO) can target each one of the three components indispensable for protein ubiquitination and subsequent degradation: the ubiquitinating machinery (E1, E2, and E3 enzymes), the protein substrate, and the proteasome (center of the circle). S-nitrosation can enhance the degradation rate of the protein substrate (point 1, e.g., $\mathrm{O}^{6}$-alkylguanyl-DNA alkyltransferase, AGT) or its ubiquitination (point 2). Conversely, $S$-nitrosation can (i) inhibit ubiquitin ligase activity of several E3s, such as Parkin and XIAP (point 3), (ii) affect ubiquitination directly, by changing protein structure, as demonstrated for Bcl2 and FLIP (point 4), or indirectly, by inhibiting enzyme activities of proteins acting as positive modifiers of ubiquitination (e.g., IKK $\beta$, point 5), and (iii) directly impair protasome activity (point 6). Red ring: inhibitory effects; green ring: activating effects.

quality control, as it is responsible for the redirection of damaged/unfolded proteins towards the proteasome to be degraded. In addition, it mediates the recognition of damaged organelles, or protein aggregates, by means of adaptor proteins (i.e., p62/sequestosome) that are also implicated in autophagy-mediated degradation [48]. This further function emphasizes the paramount role of ubiquitination in cellular homeostasis, as it is at the crossroads between different degradative pathways (both proteasome and autophagy mediated). Although ubiquitination was initially shown to drive protein degradation, it is now commonly accepted that the nature of ubiquitination (monoubiquitination versus polyubiquitination), as well as the type of interubiquitin linkages in polyubiquitin chains, mediates different response in cellular processes and signaling, including antigen processing [49], apoptosis [50], cell cycle [51], DNA transcription, and repair [52].

2.2. Inhibitory Effects of S-Nitrosation. Nitric oxide can affect ubiquitin conjugation steps and proteasomal degradation of ubiquitinated proteins at different level (Figure 2). For instance, the catalytic site of ubiquitinating enzymes (E1E2-E3) contains a cysteine residue that it is now arising could be susceptible to $S$-nitrosation [53-55]. S-nitrosation has been also shown to inhibit E3 ligase activity in RING (really interesting new gene) finger motif-containing proteins, modulating in such a way the downstream signaling 
cascades. Notably, RING E3s do not have recognizable active sites that define the "canonical" enzymes. Instead, they have large binding interfaces and act as scaffold proteins bringing together the participant E2 and substrate proteins. Therefore, $S$-nitrosation might affect the interacting properties of this class of E3 ligase. One example is the RING finger E3 ligase parkin, whose mutations have been demonstrated being implicated in Parkinson's disease etiopathogenesis. $S$-nitrosation of parkin inhibits its activity [54], thereby resulting in enhanced accumulation of protein aggregates, as well as impairment of autophagy-mediated removal of damaged mitochondria (mitophagy) [2] (Figure 2). Likewise, $S$-nitrosation of the RING finger E3 ligase X-linked IAP (XIAP) has been reported to inhibit ubiquitin-mediated proteasomal degradation of caspase 3 , thereby resulting in the promotion of cell death by apoptosis [30, 55] (Figure 2).

Beside the effects on ubiquitin conjugating system, NO can directly interfere with the proteasome protein complex. In vascular smooth muscle cell, $S$-nitrosoglutathione (GSNO) exposure revealed that the $20 \mathrm{~S}$ catalytic core of the $26 \mathrm{~S}$ proteasome contains 10 cysteines which undergo $S$-nitrosation, thus resulting in the inhibition of all three catalytic activities of the complex (chymotrypsin-, trypsin-, and caspase-like) [56] (Figure 2). Since the 26S proteasome is responsible for the time-dependent degradation of cell cycle proteins (e.g., cdk2, cdk4, cyclins, and the cyclin-dependent kinase inhibitors p21 and p27) [57], the inhibition of its activity by $S$ nitrosation can affect cell cycle progression and proliferation. The effects of $S$-nitrosation-mediated modulation of protein turnover can also directly impact on target proteins, such as in the cases of the key apoptosis regulatory protein $\mathrm{Bcl} 2$ and the antiapoptotic FLICE inhibitory protein (FLIP), whose $S$-nitrosation inhibits their ubiquitination and proteasomal degradation and finally leads to apoptosis suppression [58, 59] (Figure 2). Furthermore, $S$-nitrosation may indirectly inhibit ubiquitination via the regulation of alternative posttranslational modifications, such as in the case of the NOmediated activation of nuclear factor $\kappa$-light-chain enhancer of activated B cells (NF- $\kappa \mathrm{B})$. S-nitrosation of $\mathrm{I} \kappa \mathrm{B}$ kinase $(\mathrm{IKK} \beta)$ inhibits its kinase activity, making it unable to phosphorylate the inhibitor of NF- $\kappa \mathrm{B}$ (I $\kappa \mathrm{B})$ which, in turn, does not undergo ubiquitination and degradation via the proteasome, leaving NF- $\kappa \mathrm{B}$ unable to translocate into the nucleus and to induce transcription [60, 61] (Figure 2).

2.3. Activating Effects of S-Nitrosation. While many observations argue for $S$-nitrosation being a posttranslational modification that negatively affects UPS efficiency, there is a literature supporting the hypothesis that it can also indirectly enhance ubiquitin-mediated protein degradation. In regards to this aspect, it should be reminded that polyubiquitinationmediated degradation of some proteins relies upon the conversion of $\mathrm{N}$-terminal domain-located asparagine, glutamine or cysteine residues into arginine. This is required in order to allow recognition by E3 ligases of proteins being degraded (the so called N-rule) [62]. Arginylation of the Nterminal cysteines seems to be facilitated by $S$-nitrosation thereby suggesting the involvement of this modification in the turnover of many substrates.

The propensity of a number of proteins to undergo ubiquitination after $S$-nitrosation is not so unusual. An exhaustive example is provided by the key DNA repair protein $O^{6}$-alkylguanine-DNA alkyltransferase (AGT), whose $S$ nitrosation has been reported to induce its massive proteasomal degradation and to negatively affect DNA repair [63] (Figure 2).

\section{Ubiquitin-Protein System and Protein $S$-Nitrosation in Neuromuscular Diseases}

3.1. Muscular Atrophies and Myopathies. Skeletal muscle atrophy can be defined as wasting or decrease in muscle mass owing to injury, lack of use, or disease. Muscle atrophy arises either from damage to the nerves that supply the muscles (neuromuscular disease) or disease of the muscle itself (musculoskeletal disease). The causes of atrophy rely on genetic mutations, such as in amyotrophic lateral sclerosis and muscular dystrophies, or are derived from systemic diseases, such as diabetes, cancer, and metabolic inflammation [64]. Nevertheless, the totality of atrophic conditions shares an imbalance between protein synthesis and degradation, resulting in reduced protein synthesis and increased protein breakdown, which in turn leads to reduced muscle mass and muscle fiber size. Indeed, independently of the etiology, muscle atrophies are commonly identified by the upregulation of the same set of genes, the so-called atrophyrelated genes, or atrogenes, among which atrogin-1/muscle atrophy F-box (MAFbx) and muscle RING finger 1 (MuRF1) are well documented. These genes belong to the family of E3 ligase enzymes, which are responsible for the massive protein breakdown occurring in these diseases. In skeletal muscle atrophy, the central role of the ubiquitin-proteasome pathway has been characterized through the pioneering studies on gene expression profile independently performed by the research groups of Goldberg and Glass $[65,66]$. In particular, they revealed that atrogin-1/MAFbx and MuRF1 are the two muscle-specific ubiquitin ligases upregulated in different models of muscle atrophy and responsible for the increased protein degradation via the UPS.

Another interesting common point of muscular atrophies is the occurrence of nitroxidative stress $[67,68]$; indeed, accumulation of nitrotyrosine adducts has been detected in models of disuse-induced atrophies, as well as genetic-based dystrophies [69]. In skeletal muscle, the maintenance of a fully functioning fiber requires the correct assembly of the dystrophin glycoprotein complex (DGC). It is composed by several transmembrane and peripheral accessory proteins which are highly expressed in the sarcolemma and constitute a critical link between the cytoskeleton and the extracellular matrix [70]. It has been reported that DGC participates in cell signaling through the involvement of nNOS, which is predominant muscular isoform of NOS found to be associated to the complex via the alpha-syntrophin [71]. One possible mechanism underlying the overproduction of NO in muscle cell under atrophic conditions is the dislocation 
TABLE 2: Role and targets of NO in neuromuscular dysfunctions.

\begin{tabular}{lcl}
\hline NO adduct & Protein & Reference \\
\hline S-NO Cys-3635 & Ryanodine receptor 1 & $\begin{array}{l}\text { Bellinger et al., 2009 } \\
{[76]}\end{array}$ \\
Tyr-NO? & NFkB & Suzuki et al., 2007 [71] \\
Tyr-NO? & FoxO3 & Suzuki et al., 2007 [71] \\
S-NO & Transient receptor & Yoshida et al., 2006 [85] \\
Cys-553/558 & potential cation channel & \\
S-NO & Myogenin & Martínez-Moreno et al., \\
Cys-61/65 & & 2008 [80] \\
\hline
\end{tabular}

of nNOS from the DGC underneath the sarcolemmal membrane, followed by its redistribution into the cytosol where it produces NO [72]. The majority of congenital dystrophies depends on mutations in any of the complex components [73]. Interestingly, the dislocation of nNOS occurs in many types of dystrophies, such as Duchenne muscular dystrophy [70], which is characterized by the complete ablation of dystrophin, and in autosomal recessive limb girdle muscular dystrophy (AR-LGMD), where mutations of sarcoglycan proteins seem to be the main causative events of the pathology [74]. Furthermore, dislocation of nNOS from the DGC occurs also in rat models of disuse- or denervation-induced atrophy, indicating that this mechanism could underlie, at least in part, the pathology of muscular disorders [72]. More recently, it has been also demonstrated that nNOS dislocation induces force reduction, which is typical feature of dystrophin-null mouse models, by means of still not elucidated mechanisms putatively involving tyrosine nitration and also $S$-nitrosation [75]. The first evidence of $S$-nitrosation involvement in this class of pathologies involves the $S$ nitrosation, and the subsequent hyperactivation, of the $\mathrm{Ca}^{2+}$ release channel ryanodine receptor 1 (RyR1). Such a modification leads to a chronic $\mathrm{Ca}^{2+}$ leakage from sarcoplasmatic reticulum $[76,77]$ and triggers mitochondrial fragmentation underlying muscle atrophy [78]. Moreover, NO has been reported being involved in the activation of Forkhead box $\mathrm{O}$ (FoxO) 3a transcription factor (FoxO3a). Although the molecular mechanisms underlying this process are not well established yet, the increase of intracellular NO levels within the cell seems to be capable of mediating FoxO3a activation and nuclear translocation, thereby inducing skeletal muscle atrophy by upregulating MuRF1 or atrogin-1/MAFbx $[79,80]$. In this context, it is of note to remind that also myogenin, a protein involved in myofiber differentiation and development of functional muscles, has been reported undergoing $S$ nitrosation, reasonable at the level of Cys61 and Cys65 [81]. This modification profoundly impacts on myogenin ability to bind DNA at the promoter regions to activate downstream gene expression (e.g., caveolin-3) and finally result in muscle atrophy (Table 2).

3.2. Neuropathies. Many diseases affecting muscle health and function also induce peripheral neuropathies as side effects. Indeed, denervation or peripheral nerve injuries (e.g., those characterized by partial loss of fibers or myelin in the nerve) strongly contribute to muscle wasting [82]. Besides the already mentioned muscular dystrophies, also cancer and diabetes, as well as aging-related cachexia, show alterations of nerve physiology associated with $\mathrm{NO}$ dysbalance and PSNOs increase [72, 83]. Moreover, it has been reported that NO overproduction and $S$-nitrosation could be directly associated with the transduction pathways underlying fatigue and myalgia deriving from muscle wasting [84], which are typical features of skeletal muscle atrophic states. In particular, recent lines of evidence argue for $S$-nitrosation of the transient receptor potential vanilloid 1 and ankyrin 1 (TRPV1 and TRPA1, resp.), two polymodal ion channels of peripheral sensory dorsal root ganglia, being the principal event underlying the sensitivity of noxious stimuli impinging on peripheral nociceptors $[85,86]$ (Table 2 ).

As above reported for correct muscle maintenance, a balanced ratio between protein synthesis and degradation is important also for neuronal viability. Actually, an efficient removal of unfolded or damaged proteins and organelles is crucial to prevent neuronal death and to preserve axonal integrity. In regard to this aspect, several proteins have been reported playing a pivotal role in neurodegenerative diseases when $S$-nitrosated. Drpl is a case in point, as its mitochondrial translocation and GTPase activity seem to be enhanced when the protein undergoes S-nitrosation at Cys644 [87]. This gain-of-function modification-which has been found associated with Alzheimer's disease and pathological conditions affecting central nervous system (Table 1)-alters mitochondrial dynamics process by increasing mitochondrial fragmentation and finally contributes to neuronal cell demise. We readily refer to other comprehensive and more focused reviews dissecting in detail this aspect [88], while attempting here to deal with how $S$-nitrosation of proteins involved in ubiquitination process can impact on peripheral nervous system physiology. Among them, the ubiquitin E3 ligase TRIM2 (tripartite motif containing protein 2), which is involved in the regulation of axonal specification and polarization [89], has been very recently proposed to be neuroprotective [90]. In particular, Ylikallio and colleagues reported that TRIM2 mutations that result in the complete loss of the protein are associated with childhood onset of axonal neuropathy leading to muscle mass reduction. Mouse models of TRIM2 deficiency recapitulate the human phenotype due to an aberrant axonal accumulation of neurofilaments that are no more ubiquitinated and degraded via the proteasome [91]. Although no evidence on possible redox reactions, namely, $S$ nitrosation, have been provided yet on TRIM2, it is plausible that its occurrence could inhibit TRIM2 activity, as already demonstrated for many other members of the ubiquitin E3 ligase superfamily, thereby allowing speculating that the existence of an $S$-nitrosated form of TRIM2 could correlate with the onset of axonopathy and muscle atrophy-associated peripheral neuropathy. Likewise, the mitochondrial ubiquitin E3 ligase MITOL has been demonstrated to regulate mitochondrial dynamics, as well as to counteract the toxicity of polyglutamine-containing protein ataxin 3 [92] and mutant superoxide dismutase 1 [93], which are the main causes of Machade-Joseph disease and amyotrophic lateral sclerosis, respectively. Very recently it has been indicated that MITOL 
undergoes $S$-nitrosation and loss of activity [94], thereby resulting in mitochondrial aggregation and neuronal cell death. Among the large amount of substrates, MITOL also regulates the turnover of microtubule-associated protein $1 \mathrm{~B}$ light chain 1 (LC1) that, intriguingly, is ubiquitinated by MITOL and then subjected to proteasome-mediated degradation only when S-nitrosated [94]. Thus modified, indeed, LC1 translocates to the cytoskeleton, stabilizes microtubules and, consequently, freezes organelle transport. Therefore, under moderate (physiological) NO concentration, MITOL is required to maintain intracellular traffic by promoting $\mathrm{LC1}$ degradation. Conversely, under nitrosative/toxic conditions, such as upon $\mathrm{N}$-methyl-d-aspartate (NMDA) rector chronic activation, MITOL is inactivated, resulting in LC1 accumulation and mitochondrial dysfunction typical of neurodegenerative disease [94].

\section{Future Research Perspectives and Therapeutic Strategies}

On the basis of what previously described, excessive $S$ nitrosation seems to play a detrimental role in neurological disorders mostly due to its direct inhibitory effect on ubiquitin E3 ligases involved in the maintenance of cellular homeostasis (e.g., MITOL and TRIM2). Moreover nitrosative stress has been indicated to negatively affect muscle function and to induce muscular atrophy, owing to an excessive activation of the UPS (e.g., by means of atrogene induction via FoxO). In accordance with the above reported results, $S$ nitrosation has been also demonstrated being deeply implicated in sensitivity to nociceptive stimuli due to its impact on TRP ion channels. Altogether, these observations correlate with recent lines of evidence indicating that the sulfhydrylcontaining molecule $N$-acetylcysteine (NAC) reduces pain and ameliorates muscle performance $[95,96]$, protects dystrophic myofibers against eccentric muscle damage, and contrasts abnormal calcium influx [97]. Being NAC a wellknown antioxidant and denitrosating agent, this evidence suggests that nitrosative stress might represent a condition underlying or contributing to some pathological features of skeletal muscle disorders. Along this line, it has been demonstrated that pharmacological inhibition or genetic ablation of nNOS [75] reverts neuromuscular pathological phenotypes; however, these approaches have still not allowed discriminating whether tyrosine nitration or cysteine $S$ nitrosation is the principal mediator of neuropathy and myopathy induced by NO overproduction. Undoubtedly, the use of different NO donors does not represent a good model to unravel this issue. Indeed, their delivery of NO, which recapitulates a burst more than a persistent, and physiological, flux, has so far produced still questionable results. Cellular and mouse models of "genetically altered" S-nitrosation (e.g., GSNOR downregulating or knock-out models) could be of help in the next future to evaluate the specific contribution of different NO-mediated protein modifications: nitrationversus $S$-nitrosation. Figuring out this issue would open new avenues for the pharmacological treatment aimed at the restoration of a correct neuromuscular physiology for pathologies whose prognosis, on the contrary, is characterized by a progressive and irreversible loss of motion and cognitive abilities accompanied by chronic pain.

\section{Conflict of Interests}

The authors declare that there is no conflict of interests regarding the publication of this paper.

\section{Authors' Contribution}

Salvatore Rizza and Costanza Montagna equally contributed to this work.

\section{Acknowledgments}

This work has been partially supported by grants from the National Ministry of Health, Young Italian Researcher Grant, 2008 (Grant no. GR-2008-1138121), and from the Italian Association for Cancer Research, AIRC-MFAG 2011 (Grant no. 11452).

\section{References}

[1] F. Q. Schafer and G. R. Buettner, "Redox environment of the cell as viewed through the redox state of the glutathione disulfide/glutathione couple," Free Radical Biology and Medicine, vol. 30, no. 11, pp. 1191-1212, 2001.

[2] G. Di Giacomo, S. Rizza, C. Montagna, and G. Filomeni, "Established principles and emerging concepts on the interplay between mitochondrial physiology and S-(de)nitrosylation: implications in cancer and neurodegeneration," International Journa of Cell Biology, vol. 2012, Article ID 361872, 20 pages, 2012.

[3] Z. Gu, M. Kaul, B. Yan et al., "S-nitrosylation of matrix metalloproteinases: signaling pathway to neuronal cell death," Science, vol. 297, no. 5584, pp. 1186-1190, 2002.

[4] M. A. Marietta, "Nitric oxide synthase: aspects concerning structure and catalysis," Cell, vol. 78, no. 6, pp. 927-930, 1994.

[5] O. W. Griffith and D. J. Stuehr, "Nitric oxide synthases: properties and catalytic mechanism," Annual Review of Physiology, vol. 57, pp. 707-736, 1995.

[6] R. M. Rapoport, M. B. Draznin, and F. Murad, "Endotheliumdependent relaxation in rat aorta may be mediated through cyclic GMP-dependent protein phosphorylation," Nature, vol. 306, no. 5939, pp. 174-176, 1983.

[7] R. M. J. Palmer, A. G. Ferrige, and S. Moncada, "Nitric oxide release accounts for the biological activity of endotheliumderived relaxing factor," Nature, vol. 327, no. 6122, pp. 524-526, 1987.

[8] C. Bogdan, "Nitric oxide and the immune response," Nature Immunology, vol. 2, no. 10, pp. 907-916, 2001.

[9] J. R. Steinert, S. W. Robinson, H. Tong, M. D. Haustein, C. KoppScheinpflug, and I. D. Forsythe, "Nitric oxide is an activitydependent regulator of target neuron intrinsic excitability," Neuron, vol. 71, no. 2, pp. 291-305, 2011.

[10] G. C. Brown, "Regulation of mitochondrial respiration by nitric oxide inhibition of cytochrome c oxidase," Biochimica et Biophysica Acta, vol. 1504, no. 1, pp. 46-57, 2001. 
[11] J. P. Collman, A. Dey, R. A. Decreau et al., "Interaction of nitric oxide with a functional model of cytochrome c oxidase," Proceedings of the National Academy of Sciences of the United States of America, vol. 105, no. 29, pp. 9892-9896, 2008.

[12] M. G. Mason, P. Nicholls, M. T. Wilson, and C. E. Cooper, "Nitric oxide inhibition of respiration involves both competitive (heme) and noncompetitive (copper) binding to cytochrome c oxidase," Proceedings of the National Academy of Sciences of the United States of America, vol. 103, no. 3, pp. 708-713, 2006.

[13] D. T. Hess, A. Matsumoto, S.-O. Kim, H. E. Marshall, and J. S. Stamler, "Protein S-nitrosylation: purview and parameters," Nature Reviews Molecular Cell Biology, vol. 6, no. 2, pp. 150-166, 2005.

[14] A. Martínez-Ruiz and S. Lamas, "S-nitrosylation: a potential new paradigm in signal transduction," Cardiovascular Research, vol. 62, no. 1, pp. 43-52, 2004.

[15] M.-C. Broillet, "S-nitrosylation of proteins," Cellular and Molecular Life Sciences, vol. 55, no. 8-9, pp. 1036-1042, 1999.

[16] L. Liu, A. Hausladen, M. Zeng, L. Que, J. Heitman, and J. S. Stamler, "A metabolic enzyme for S-nitrosothiol conserved from bacteria to humans," Nature, vol. 410, no. 6827, pp. 490494, 2001.

[17] J. B. Mannick, A. Hausladen, L. Liu et al., "Fas-induced caspase denitrosylation," Science, vol. 284, no. 5414, pp. 651-654, 1999.

[18] M. Benhar, M. T. Forrester, and J. S. Stamler, "Protein denitrosylation: enzymatic mechanisms and cellular functions," Nature Reviews Molecular Cell Biology, vol. 10, no. 10, pp. 721-732, 2009.

[19] R. Radi, "Nitric oxide, oxidants, and protein tyrosinenitration," Proceedings of the National Academy of Sciences of the USA, vol. 101, no. 12, pp. 4003-4008, 2004.

[20] J. S. Beckman, "Oxidative damage and tyrosine nitration from peroxynitrite," Chemical Research in Toxicology, vol. 9, no. 5, pp. 836-844, 1996.

[21] J. S. Stamler, S. Lamas, and F. C. Fang, "Nitrosylation: the prototypic redox-based signaling mechanism," Cell, vol. 106, no. 6, pp. 675-683, 2001.

[22] J. S. Stamler, E. J. Toone, S. A. Lipton, and N. J. Sucher, "(S)NO signals: translocation, regulation, and a consensus motif," Neuron, vol. 18, no. 5, pp. 691-696, 1997.

[23] S. M. Marino and V. N. Gladyshev, "Structural analysis of cysteine S-nitrosylation: a modified acid-based motif and the emerging role of trans-nitrosylation," Journal of Molecular Biology, vol. 395, no. 4, pp. 844-859, 2010.

[24] G. P. H. Ho, B. Selvakumar, J. Mukai et al., "S-nitrosylation and S-palmitoylation reciprocally regulate synaptic targeting of PSD-95," Neuron, vol. 71, no. 1, pp. 131-141, 2011.

[25] T. Nakamura and S. A. Lipton, "Emerging role of proteinprotein transnitrosylation in cell signaling pathways," Antioxidants and Redox Signaling, vol. 18, no. 3, pp. 239-249, 2013.

[26] C. Wu, T. Liu, W. Chen et al., "Redox regulatory mechanism of transnitrosylation by thioredoxin," Molecular and Cellular Proteomics, vol. 9, no. 10, pp. 2262-2275, 2010.

[27] J. R. Pawloski, D. T. Hes, and J. S. Stamler, "Export by red blood cells of nitric oxide bioactivity," Nature, vol. 409, no. 6820, pp. 622-626, 2001.

[28] M. Benhar, M. T. Forrester, D. T. Hess, and J. S. Stamler, "Regulated protein denitrosylation by cytosolic and mitochondrial thioredoxins," Science, vol. 320, no. 5879, pp. 1050-1054, 2008.

[29] Y. Suzuki, Y. Nakabayashi, and R. Takahashi, "Ubiquitinprotein ligase activity of $\mathrm{X}$-linked inhibitor of apoptosis protein promotes proteasomal degradation of caspase- 3 and enhances its anti-apoptotic effect in Fas-induced cell death," Proceedings of the National Academy of Sciences of the United States of America, vol. 98, no. 15, pp. 8662-8667, 2001.

[30] T. Nakamura, L. Wang, C. C. L. Wong et al., "Transnitrosylation of XIAP regulates caspase-dependent neuronal cell death," Molecular Cell, vol. 39, no. 2, pp. 184-195, 2010.

[31] M. D. Kornberg, N. Sen, M. R. Hara et al., "GAPDH mediates nitrosylation of nuclear proteins," Nature Cell Biology, vol. 12, no. 11, pp. 1094-1100, 2010.

[32] A. Sawa, A. A. Khan, L. D. Hester, and S. H. Snyder, "Glyceraldehyde-3-phosphate dehydrogenase: nuclear translocation participates in neuronal and nonneuronal cell death," Proceedings of the National Academy of Sciences of the United States of America, vol. 94, no. 21, pp. 11669-11674, 1997.

[33] K. Meuer, I. E. Suppanz, P. Lingor et al., "Cyclin-dependent kinase 5 is an upstream regulator of mitochondrial fission during neuronal apoptosis," Cell Death and Differentiation, vol. 14 , no. 4, pp. 651-661, 2007.

[34] A. Holmgren, "Biochemistry: SNO removal," Science, vol. 320, no. 5879, pp. 1019-1020, 2008.

[35] R. Sengupta, S. W. Ryter, B. S. Zuckerbraun, E. Tzeng, T. R. Billiar, and D. A. Stoyanovsky, "Thioredoxin catalyzes the denitrosation of low-molecular mass and protein S-nitrosothiols," Biochemistry, vol. 46, no. 28, pp. 8472-8483, 2007.

[36] D. T. Hess and J. S. Stamler, "Regulation by S-nitrosylation of protein post-translational modification," The Journal of Biological Chemistry, vol. 287, no. 7, pp. 4411-4418, 2012.

[37] H.-S. Park, J.-W. Yu, J.-H. Cho et al., "Inhibition of apoptosis signal-regulating kinase 1 by nitric oxide through a thiol redox mechanism," The Journal of Biological Chemistry, vol. 279, no. 9, pp. 7584-7590, 2004.

[38] H.-S. Park, S.-H. Huh, M.-S. Kim, S. H. Lee, and E.-J. Choi, "Nitric oxide negatively regulates c-Jun N-terminal kinase/stress-activated protein kinase by means of Snitrosylation," Proceedings of the National Academy of Sciences of the United States of America, vol. 97, no. 26, pp. 14382-14387, 2000.

[39] H.-S. Park, J.-S. Mo, and E.-J. Choi, "Nitric oxide inhibits an interaction between JNK1 and c-Jun through nitrosylation," Biochemical and Biophysical Research Communications, vol. 351, no. 1, pp. 281-286, 2006.

[40] G. Filomeni, G. Rotilio, and M. R. Ciriolo, "Disulfide relays and phosphorylative cascades: partners in redox-mediated signaling pathways," Cell Death and Differentiation, vol. 12, no. 12, pp. 1555-1563, 2005.

[41] A. Nott, P. M. Watson, J. D. Robinson, L. Crepaldi, and A. Riccio, "S-nitrosylation of histone deacetylase 2 induces chromatin remodelling in neurons," Nature, vol. 455, no. 7211, pp. 411-415, 2008.

[42] M. Obin, F. Shang, X. Gong, G. Handelman, J. Blumberg, and A. Taylor, "Redox regulation of ubiquitin-conjugating enzymes: mechanistic insights using the thiol-specific oxidant diamide," FASEB Journal, vol. 12, no. 7, pp. 561-569, 1998.

[43] J. Jahngen-Hodge, M. S. Obin, X. Gong et al., "Regulation of ubiquitin-conjugating enzymes by glutathione following oxidative stress," The Journal of Biological Chemistry, vol. 272, no. 45, pp. 28218-28226, 1997.

[44] K. S. Doris, E. L. Rumsby, and B. A. Morgan, "Oxidative stress responses involve oxidation of a conserved ubiquitin pathway enzyme," Molecular and Cellular Biology, vol. 32, no. 21, pp. 4472-4481, 2012. 
[45] G. Bossis and F. Melchior, "Regulation of SUMOylation by reversible oxidation of SUMO conjugating enzymes," Molecular Cell, vol. 21, no. 3, pp. 349-357, 2006.

[46] A. Ciechanover, "The ubiquitin-proteasome proteolytic pathway," Cell, vol. 79, no. 1, pp. 13-21, 1994.

[47] V. Kirkin, D. G. McEwan, I. Novak, and I. Dikic, "A role for ubiquitin in selective autophagy," Molecular Cell, vol. 34, no. 3, pp. 259-269, 2009.

[48] A. L. Goldberg and K. L. Rock, "Proteolysis, proteasomes and antigen presentation," Nature, vol. 357, no. 6377, pp. 375-379, 1992.

[49] D. Vucic, V. M. Dixit, and I. E. Wertz, "Ubiquitylation in apoptosis: a post-translational modification at the edge of life and death," Nature Reviews Molecular Cell Biology, vol. 12, no. 7, pp. 439-452, 2011.

[50] A. Mocciaro and M. Rape, "Emerging regulatory mechanisms in ubiquitindependent cell cycle control," Journal of Cell Science, vol. 125, no. 2, pp. 255-263, 2012.

[51] D. E. Wright, C.-Y. Wang, and C.-F. Kao, "Histone ubiquitylation and chromatin dynamics," Frontiers in Bioscience, vol. 17, no. 3, pp. 1051-1078, 2012.

[52] T. Nakamura and S. A. Lipton, "S-nitrosylation of critical protein thiols mediates protein misfolding and mitochondrial dysfunction in neurodegenerative diseases," Antioxidants and Redox Signaling, vol. 14, no. 8, pp. 1479-1492, 2011.

[53] K. K. K. Chung, B. Thomas, X. Li et al., "S-nitrosylation of parkin regulates ubiquitination and compromises parkin's protective function," Science, vol. 304, no. 5675, pp. 1328-1331, 2004.

[54] A. H. K. Tsang, Y.-I. L. Lee, H. S. Ko et al., "S-nitrosylation of XIAP compromises neuronal survival in Parkinson's disease," Proceedings of the National Academy of Sciences of the United States of America, vol. 106, no. 12, pp. 4900-4905, 2009.

[55] M. R. Kapadia, J. W. Eng, Q. Jiang, D. A. Stoyanovsky, and M. R. Kibbe, "Nitric oxide regulates the $26 \mathrm{~S}$ proteasome in vascular smooth muscle cells," Nitric Oxide, vol. 20, no. 4, pp. 279-288, 2009.

[56] A. J. Obaya and J. M. Sedivy, "Regulation of cyclin-Cdk activity in mammalian cells," Cellular and Molecular Life Sciences, vol. 59, no. 1, pp. 126-142, 2002.

[57] N. Azad, V. Vallyathan, L. Wang et al., "S-nitrosylation of Bcl2 inhibits its ubiquitin-proteasomal degradation: a novel antiapoptotic mechanism that suppresses apoptosis," The Journal of Biological Chemistry, vol. 281, no. 45, pp. 34124-34134, 2006.

[58] P. Chanvorachote, U. Nimmannit, L. Wang et al., "Nitric oxide negatively regulates Fas CD95-induced apoptosis through inhibition of ubiquitin-proteasome-mediated degradation of FLICE inhibitory protein," The Journal of Biological Chemistry, vol. 280, no. 51, pp. 42044-42050, 2005.

[59] R.-G. Hu, J. Sheng, X. Qi, Z. Xu, T. T. Takahashi, and A. Varshavsky, "The N-end rule pathway as a nitric oxide sensor controlling the levels of multiple regulators," Nature, vol. 437, no. 7061, pp. 981-986, 2005.

[60] N. L. Reynaert, K. Ckless, S. H. Korn et al., "Nitric oxide represses inhibitory $\kappa \mathrm{B}$ kinase through S-nitrosylation," Proceedings of the National Academy of Sciences of the United States of America, vol. 101, no. 24, pp. 8945-8950, 2004.

[61] D. J. Glass, "Molecular mechanisms modulating muscle mass," Trends in Molecular Medicine, vol. 9, no. 8, pp. 344-350, 2003.

[62] W. Wei, B. Li, M. A. Hanes, S. Kakar, X. Chen, and L. Liu, "S-nitrosylation from GSNOR deficiency impairs DNA repair and promotes hepatocarcinogenesis," Science Translational Medicine, vol. 2, no. 19, Article ID 19ra13, 2010.

[63] M. Karin and Y. Ben-Neriah, "Phosphorylation meets ubiquitination: the control of NF- $\kappa \mathrm{B}$ activity," Annual Review of Immunology, vol. 18, pp. 621-663, 2000.

[64] S. C. Bodine, E. Latres, S. Baumhueter et al., "Identification of ubiquitin ligases required for skeletal Muscle Atrophy," Science, vol. 294, no. 5547, pp. 1704-1708, 2001.

[65] M. D. Gomes, S. H. Lecker, R. T. Jagoe, A. Navon, and A. L. Goldberg, "Atrogin-1, a muscle-specific F-box protein highly expressed during muscle atrophy," Proceedings of the National Academy of Sciences of the United States of America, vol. 98, no. 25, pp. 14440-14445, 2001.

[66] H. C. Lehmann, A. Köhne, G. M. Zu Hörste et al., "Role of nitric oxide as mediator of nerve injury in inflammatory neuropathies," Journal of Neuropathology and Experimental Neurology, vol. 66, no. 4, pp. 305-312, 2007.

[67] J. M. Souza, G. Peluffo, and R. Radi, "Protein tyrosine nitrationFunctional alteration or just a biomarker?" Free Radical Biology and Medicine, vol. 45, no. 4, pp. 357-366, 2008.

[68] R. W. R. Dudley, G. Danialou, K. Govindaraju, L. Lands, D. E. Eidelman, and B. J. Petrof, "Sarcolemmal damage in dystrophin deficiency is modulated by synergistic interactions between mechanical and oxidative/nitrosative stresses," The American Journal of Pathology, vol. 168, no. 4, pp. 1276-1287, 2006.

[69] J. E. Brenman, D. S. Chao, H. Xia, K. Aldape, and D. S. Bredt, "Nitric oxide synthase complexed with dystrophin and absent from skeletal muscle sarcolemma in Duchenne muscular dystrophy," Cell, vol. 82, no. 5, pp. 743-752, 1995.

[70] Z. Grozdanovic, "NO message from muscle," Microscopy Research and Technique, vol. 55, no. 3, pp. 148-153, 2001.

[71] N. Suzuki, N. Motohashi, A. Uezumi et al., "NO production results in suspension-induced muscle atrophy through dislocation of neuronal NOS," Journal of Clinical Investigation, vol. 117, no. 9, pp. 2468-2476, 2007.

[72] T. S. Khurana and K. E. Davies, "Pharmacological strategies for muscular dystrophy," Nature Reviews Drug Discovery, vol. 2, no. 5, pp. 379-390, 2003.

[73] R. H. Crosbie, R. Barresi, and K. P. Campbell, "Loss of sarcolemma nNOS in sarcoglycan-deficient muscle," FASEB Journal, vol. 16, no. 13, pp. 1786-1791, 2002.

[74] D. Li, Y. Yue, Y. Lai, C. H. Hakim, and D. Duan, "Nitrosative stress elicited by nNOS $\mu$ delocalization inhibits muscle force in dystrophin-null mice," Journal of Pathology, vol. 223, no. 1, pp. 88-98, 2011.

[75] J. Sun, C. Xin, J. P. Eu, J. S. Stamler, and G. Meissner, "Cysteine3635 is responsible for skeletal muscle ryanodine receptor modulation by NO," Proceedings of the National Academy of Sciences of the United States of America, vol. 98, no. 20, pp. 11158$11162,2001$.

[76] A. M. Bellinger, S. Reiken, C. Carlson et al., "Hypernitrosylated ryanodine receptor calcium release channels are leaky in dystrophic muscle," Nature Medicine, vol. 15, no. 3, pp. 325-330, 2009.

[77] Z. Gu, T. Nakamura, and S. A. Lipton, "Redox reactions induced by nitrosative stress mediate protein misfolding and mitochondrial dysfunction in neurodegenerative diseases," Molecular Neurobiology, vol. 41, no. 2-3, pp. 55-72, 2010.

[78] M. Sandri, C. Sandri, A. Gilbert et al., "Foxo transcription factors induce the atrophy-related ubiquitin ligase atrogin-1 and cause skeletal muscle atrophy," Cell, vol. 117, no. 3, pp. 399-412, 2004 . 
[79] S. H. Lecker, R. T. Jagoe, A. Gilbert et al., "Multiple types of skeletal muscle atrophy involve a common program of changes in gene expression," FASEB Journal, vol. 18, no. 1, pp. 39-51, 2004.

[80] M. Martínez-Moreno, A. Martínez-Ruiz, A. Álvarez-Barrientos, F. Gavilanes, S. Lamas, and I. Rodríguez-Crespo, "Nitric oxide down-regulates caveolin-3 levels through the interaction with myogenin, its transcription factor," The Journal of Biological Chemistry, vol. 282, no. 32, pp. 23044-23054, 2007.

[81] R. R. Kohn, "Denervation muscle atrophy: an autolytic system in vitro," The American Journal of Pathology, vol. 47, pp. 315-323, 1965.

[82] M. Kaneki, N. Shimizu, D. Yamada, and K. Chang, "Nitrosative stress and pathogenesis of insulin resistance," Antioxidants and Redox Signaling, vol. 9, no. 3, pp. 319-329, 2007.

[83] M.-A. Choe and G. J. An, "Effects of nitric oxide synthase inhibitor on hindlimb muscles in rats with neuropathic pain induced by unilateral peripheral nerve injury," Journal of Korean Academy of Nursing, vol. 41, no. 4, pp. 520-527, 2011.

[84] T. Miyamoto, A. E. Dublin, M. J. Petrus, and A. Patapoutian, "TRPV1 and TRPA1 mediate peripheral nitric oxide-induced nociception in mice," PLoS ONE, vol. 4, no. 10, Article ID e7596, 2009.

[85] T. Yoshida, R. Inoue, T. Morii et al., "Nitric oxide activates TRP channels by cysteine S-nitrosylation," Nature Chemical Biology, vol. 2, no. 11, pp. 596-607, 2006.

[86] D.-H. Cho, T. Nakamura, J. Fang et al., " $\beta$-Amyloid-related mitochondrial fission and neuronal injury," Science, vol. 324, no. 5923, pp. 102-105, 2009.

[87] T. Nakamura and S. A. Lipton, "Redox regulation of mitochondrial fission, protein misfolding, synaptic damage, and neuronal cell death: potential implications for Alzheimer's and Parkinson's diseases," Apoptosis, vol. 15, no. 11, pp. 1354-1363, 2010.

[88] M. R. Khazaei, E. C. Bunk, A.-L. Hillje et al., "The E3-ubiquitin ligase TRIM2 regulates neuronal polarization," Journal of Neurochemistry, vol. 117, no. 1, pp. 29-37, 2011.

[89] E. Ylikallio, R. Poyhonen, M. Zimon et al., "Deficiency of the E3 ubiquitin ligase TRIM2 in early-onset axonal neuropathy," Human Molecular Genetics, vol. 22, no. 15, pp. 2975-2983, 2013.

[90] M. Balastik, F. Ferraguti, A. Pires-da Silva et al., "Deficiency in ubiquitin ligase TRIM2 causes accumulation of neurofilament light chain and neurodegeneration," Proceedings of the National Academy of Sciences of the United States of America, vol. 105, no. 33, pp. 12016-12021, 2008.

[91] A. Sugiura, R. Yonashiro, T. Fukuda et al., "A mitochondrial ubiquitin ligase MITOL controls cell toxicity of polyglutamineexpanded protein," Mitochondrion, vol. 11, no. 1, pp. 139-146, 2011.

[92] R. Yonashiro, A. Sugiura, M. Miyachi et al., "Mitochondrial ubiquitin ligase MITOL ubiquitinates mutant SOD1 and attenuates mutant SOD1-induced reactive oxygen species generation," Molecular Biology of the Cell, vol. 20, no. 21, pp. 4524-4530, 2009.

[93] R. Yonashiro, Y. Kimijima, T. Shimura et al., "Mitochondrial ubiquitin ligase MITOL blocks S-nitrosylated MAP1B-light chain 1-mediated mitochondrial dysfunction and neuronal cell death," Proceedings of the National Academy of Sciences of the United States of America, vol. 109, no. 7, pp. 2382-2387, 2012.

[94] M. B. Reid, D. S. Stokić, S. M. Koch, F. A. Khawli, and A. A. Leis, "N-acetylcysteine inhibits muscle fatigue in humans," Journal of Clinical Investigation, vol. 94, no. 6, pp. 2468-2474, 1994.
[95] J. S. Stamler, Q.-A. Sun, and D. T. Hess, "A SNO storm in skeletal muscle," Cell, vol. 133, no. 1, pp. 33-35, 2008.

[96] N. P. Whitehead, C. Pham, O. L. Gervasio, and D. G. Allen, "NAcetylcysteine ameliorates skeletal muscle pathophysiology in mdx mice," Journal of Physiology, vol. 586, no. 7, pp. 2003-2014, 2008.

[97] J. Fang, T. Nakamura, D.-H. Cho, Z. Gu, and S. A. Lipton, "S-nitrosylation of peroxiredoxin 2 promotes oxidative stressinduced neuronal cell death in Parkinson's disease," Proceedings of the National Academy of Sciences of the United States of America, vol. 104, no. 47, pp. 18742-18747, 2007.

[98] T. Uehara, T. Nakamura, D. Yao et al., "S-Nitrosylated proteindisulphide isomerase links protein misfolding to neurodegeneration," Nature, vol. 441, no. 7092, pp. 513-517, 2006.

[99] D. R. Gonzalez, F. Beigi, A. V. Treuer, and J. M. Hare, "Deficient ryanodine receptor S-nitrosylation increases sarcoplasmic reticulum calcium leak and arrhythmogenesis in cardiomyocytes," Proceedings of the National Academy of Sciences of the United States of America, vol. 104, no. 51, pp. 20612-20617, 2007.

[100] D. C. Andersson, A. C. Meli, S. Reiken et al., "Leaky ryanodine receptors in $\beta$-sarcoglycan deficient mice: a potential common defect in muscular dystrophy," Skeletal Muscle, vol. 2, no. 1, p. 9, 2012. 

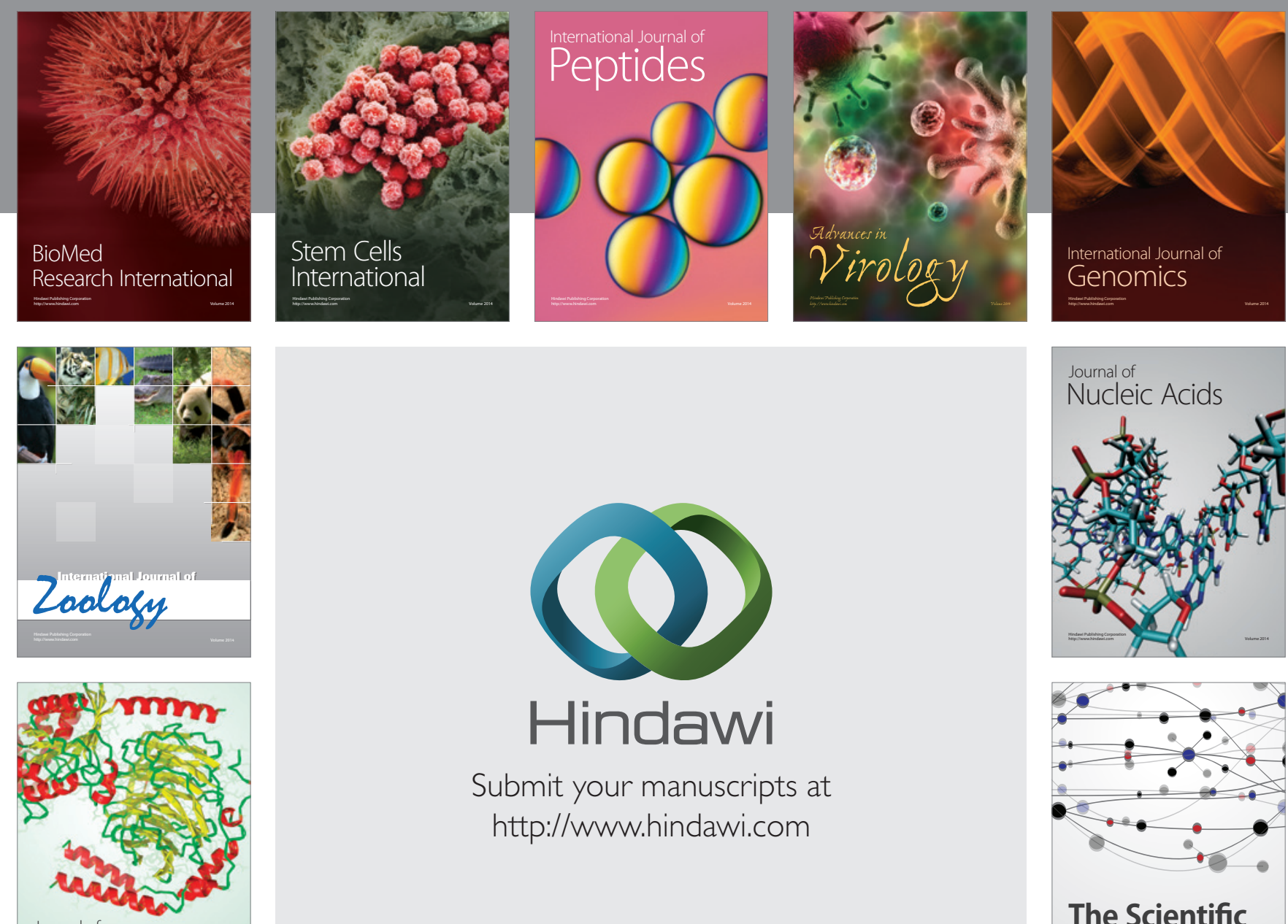

Submit your manuscripts at

http://www.hindawi.com

Journal of
Signal Transduction
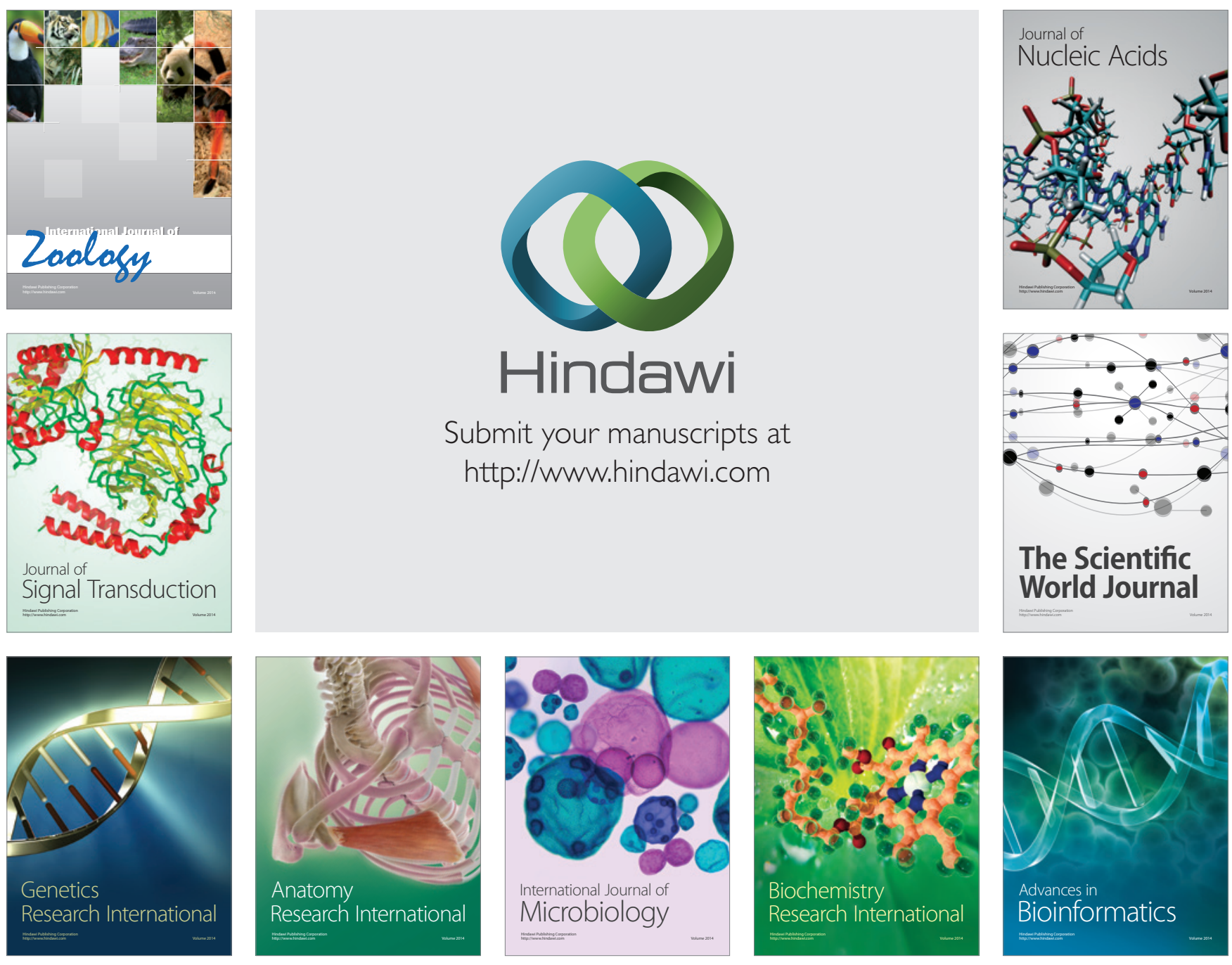

The Scientific World Journal
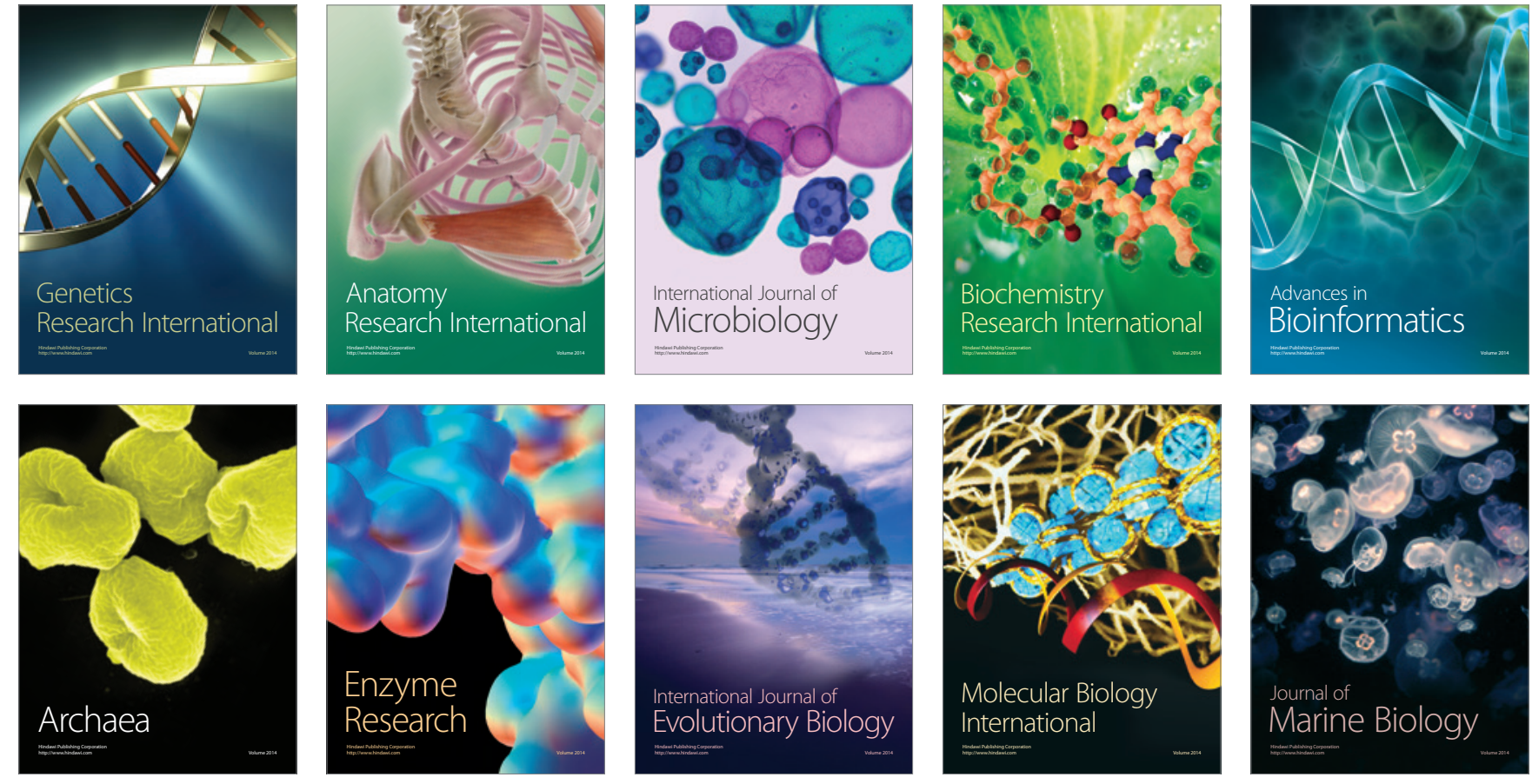